\title{
Elevada abundância de drosofilídeos e baixa riqueza de espécies, uma realidade da composição em ambiente cultivado de Pernambuco
}

\author{
High abundance of drosophilids and low species richness, a reality of composition in a cultivated \\ environment in Pernambuco
}

Gran abundancia de drosofílidos y baja riqueza de especies, una realidad de composición en un medio cultivado en Pernambuco

\author{
Merodaque da Silva Salvino \\ ORCID: https://orcid.org/0000-0002-4574-7525 \\ Universidade de Pernambuco, Brasil \\ E-mail: merodaque.9@ hotmail.com \\ Claudia Rohde \\ ORCID: https://orcid.org/0000-0002-0977-9239 \\ Universidade Federal de Pernambuco, Brasil \\ E-mail:claudiaufpe@gmail.com \\ Cícero Jorge Verçosa \\ ORCID: https://orcid.org/0000-0002-3284-6719 \\ Secretaria de Educação e Esportes de Pernambuco, Brasil \\ E-mail: cjvercosa@hotmail.com \\ Janaína Freire Clementino Mendes \\ ORCID: https://orcid.org/0000-0003-2453-4325 \\ Secretaria de Educação e Esportes de Pernambuco, Brasil \\ E-mail: janainamendes86@gmail.com \\ Rita Dayane Coutinho da Silva \\ ORCID: https://orcid.org/0000-0003-3689-8003 \\ Secretaria de Educação e Esportes de Pernambuco, Brasil \\ E-mail: rita_dayane@hotmail.com \\ Georgia Fernanda Oliveira \\ ORCID: https://orcid.org/0000-0002-9298-0987 \\ Secretaria de Educação e Esportes de Pernambuco, Brasil \\ E-mail: georgia.fernanda@gmail.com
}

\begin{abstract}
Resumo
Os drosofilídeos são organismos que se adaptam aos mais variados ambientes, naturais preservados ou antropizados. Os impactos causados em áreas naturais interferem diretamente na composição das diferentes espécies que colonizam este ambiente. Por apresentar sensibilidade às variações ambientais, essas pequenas moscas vêm sendo utilizadas como bioindicadoras naturais de alterações ambientais, enquanto outras possuem grande poder de dispersão e colonização em diferentes ecossistemas. Alterações ambientais podem interferir na abundância das espécies presentes no ambiente natural, ocasionando a extinção local de alguma destas espécies. Entre os biomas brasileiros, a Floresta Atlântica é o mais destruído pelas ações antrópicas, sendo, por isso, considerada uma das prioridades mundiais para a conservação da biodiversidade. Com a finalidade de melhor compreender os efeitos desse processo e caracterizar a composição de Drosophilidae (Insecta, Diptera) em um ambiente devastado de Floresta Atlântica, considerada área de cultivo no Município de Nazaré da Mata, Pernambuco, esses insetos foram coletados em dois períodos sazonais distintos (chuvoso e seco). Foram amostrados um total de 5524 indivíduos, sendo 3405 no período chuvoso e 2119 no período seco, representados por quatro (Drosophila, Rhinoleuconphenga, Sacaptodrosofila e Zaprionus) e 16 espécies de drosofílideos, dos quais o gênero Drosophila apresentou maior representatividade, com 13 espécies reconhecidas. Contabilizando ambas as coletas, houve uma grande predominância de três espécies exóticas: Drosophila malerkotliana, D. simulans e Zaprionus indianus, que representam $78 \%$ do total de indivíduos. A análise da riqueza (Taxa S) foi similar entre os períodos amostrados, entretanto, a maior abundância de indivíduos foi registrada no período das chuvas. Os resultados de análise de diversidade de Simpson (1-D) e Shannon (H) apontam que não houve diferença significativa para os períodos sazonais quanto à fauna de drosofílídeos.
\end{abstract}

Palavras-chave: Floresta Atlântica; Área cultivada; Insetos; Drosofilídeos.

\section{Abstract}

Drosophilids are organisms that adapt to the most varied environments, natural preserved or anthropized. The impacts caused in natural areas directly interfere in the composition of the different species that colonize this environment. 
Due to their sensitivity to environmental variations, these small flies have been used as natural bioindicators of environmental changes, while others have great dispersal and colonization power in different ecosystems. Environmental changes can interfere with the abundance of species present in the natural environment, causing the local extinction of some of these species. Among the Brazilian biomes, the Atlantic Forest is the most destroyed by anthropic actions, being, therefore, considered one of the world priorities for the conservation of biodiversity. In order to better understand the effects of this process and characterize the composition of Drosophilidae (Insecta, Diptera) in a devastated Atlantic Forest environment, considered a cultivation area in the Municipality of Nazaré da Mata, Pernambuco, these insects were collected in two seasonal periods distinct (rainy and dry). A total of 5524 individuals were sampled, 3405 in the rainy season and 2119 in the dry season, represented by four (Drosophila, Rhinoleuconphenga, Sacaptodrosophila and Zaprionus) and 16 species of Drosophila, of which the genus Drosophila was more representative, with 13 recognized species. Accounting for both collections, there was a great predominance of three exotic species: Drosophila malerkotliana, D. simulans and Zaprionus indianus, which represent $78 \%$ of the total number of individuals. The analysis of richness (Taxa S) was similar between the sampled periods, however, the greatest abundance of individuals was registered in the rainy season. The Simpson (1-D) and Shannon (H) diversity analysis results indicate that there was no significant difference for the seasonal periods regarding the drosophilid fauna.

Keywords: Atlantic Forest; Cultivated área; Insects; Drosophilids.

\section{Resumen}

Los drosófilos son organismos que se adaptan a los más variados entornos, preservados de forma natural o antropizados. Los impactos ocasionados en áreas naturales interfieren directamente en la composición de las diferentes especies que colonizan este medio. Por su sensibilidad a las variaciones ambientales, estas pequeñas moscas se han utilizado como bioindicadores naturales de cambios ambientales, mientras que otras tienen un gran poder de dispersión y colonización en diferentes ecosistemas. Los cambios ambientales pueden interferir con la abundancia de especies presentes en el medio natural, provocando la extinción local de algunas de estas especies. Entre los biomas brasileños, la Mata Atlántica es la más destruida por acciones antrópicas, por lo que es considerada una de las prioridades mundiales para la conservación de la biodiversidad. Con el fin de comprender mejor los efectos de este proceso y caracterizar la composición de Drosophilidae (Insecta, Diptera) en un ambiente devastado de Bosque Atlántico, considerado un área de cultivo en el Municipio de Nazaré da Mata, Pernambuco, estos insectos fueron recolectados en dos períodos estacionales. distinto (lluvioso y seco). Se muestrearon un total de 5524 individuos, 3405 en época de lluvias y 2119 en época seca, representados por cuatro (Drosophila, Rhinoleuconphenga, Sacaptodrosophila y Zaprionus) y 16 especies de Drosophila, de las cuales el género Drosophila fue más representativo, con 13 reconocidas. especie. Dando cuenta de ambas colecciones, hubo un gran predominio de tres especies exóticas: Drosophila malerkotliana, D. simulans y Zaprionus indianus, que representan el $78 \%$ del total de individuos. El análisis de riqueza (Tasa S) fue similar entre los períodos muestreados, sin embargo, la mayor abundancia de individuos se registró en la época de lluvias. Los resultados del análisis de diversidad de Simpson (1D) y Shannon $(\mathrm{H})$ indican que no hubo diferencia significativa para los períodos estacionales con respecto a la fauna de drosofílidos.

Palabras clave: Mata Atlántica; Superficie cultivada; Insectos; Drosophilids.

\section{Introdução}

O Brasil apresenta grande diversidade de habitats e ecossistemas, sendo a Floresta Atlântica um dos mais importantes biomas do país. Os impactos causados nesta área interferem diretamente na composição das diferentes espécies que colonizaram este ambiente. Segundo dados do Instituto Nacional de Pesquisas Espaciais (INPE), restam apenas 7,9\% de remanescentes de Floresta Atlântica em áreas acima de 100 hectares em comparação ao que havia originalmente (INPE, 2010). A Floresta Atlântica abrange cerca de $15 \%$ do território nacional, em 17 estados. É o lar de $72 \%$ dos brasileiros e concentra $70 \%$ do PIB nacional, dela dependem serviços essenciais como abastecimento de água, regulação do clima, agricultura, pesca energia elétrica e turismo. Alguns dados revelam aumento de quase 30\% no desmatamento da Floresta Atlântica, na última edição do Atlas, relativa ao período de 2018 a 2019, com remanescentes representados em apenas 12,4\% da floresta que existia originalmente (SOS Mata Atlantica, 2020).

A destruição de parcelas ainda que pequenas dessa floresta pode significar a perda irreversível de inúmeras espécies, por vezes sequer estudadas pela ciência (SOS Mata Atlântica, 2002). A eliminação das florestas nordestinas propiciou os efeitos danosos de condições climáticas potencialmente perigosas, ficando o Nordeste cada vez mais privado dos ecossistemas 
florestais protetores, que ali se adaptaram durante milhões de anos a uma faixa de condições ecológicas favoráveis (Rêgo \& Hoeflich, 2001).

A compreensão do efeito das atividades humanas sob as populações de animais, de maneira geral, é ainda bastante superficial. Sabe-se que extensas áreas com monoculturas aumentam muito a oferta de alimento para os insetos, beneficiando algumas espécies em detrimento de outras, podendo estas se tornarem pragas (Camargo, 2001). Grande parte das áreas que compreendiam Floresta Atlântica |nativa se tornaram áreas de cultivo, especialmente monoculturas. É válido compreender como a diversidade desses locais se comportam, para elucidar eventuais e necessários programas de conservação.

Entre as diversas formas de vida encontradas na Floresta Atlântica remanescente e/ou fragmentada, encontram-se os insetos. Os insetos são o grupo dominante entre os animais na Terra, no que se refere ao número de indivíduos, diversidade de espécies e sua alta capacidade de adaptação, que lhes permite ocupar praticamente todos os ambientes (Triplehorn \& Jonnson, 2011).

As pequenas moscas da família Drosophilidae (Insecta, Diptera) são insetos bem caracterizados taxonomicamente, com mais de 4 mil espécies conhecidas (Bächli, 2020). Eles ocorrem nos mais variados ambientes: áridos, úmidos e urbanos (Oliveira, Rohde, Garcia, Montes, Valente, et al 2016, Gottscalk, Hofmann, Valente, 2008; Garcia, Hochmüller, Valente, Schmitz, 2012). Exploram frutos em estado de decomposição, bem como outros substratos (Schmitz, Valente, 2019; Powel, 1997).

O objetivo deste trabalho foi monitorar a composição e diversidade de drosofilídeos em um ambiente devastado de Floresta Atlântica, considerada área de cultivo no Município de Nazaré da Mata, Pernambuco, Brasil, para entender processos básicos da biologia de populações de insetos dessa família. Trata-se de uma pesquisa de campo.

\section{Metodologia}

\section{Área de estudo}

As amostragens foram realizadas na zona rural do município de Nazaré da Mata, em uma região chamada de Engenho Morojó ( $07^{\circ} 43^{\prime} 45.6^{\prime}$ S, $035^{\circ} 16^{\prime} 35.1$ ” W, 129m de altitude). Trata-se de uma área habitada por aproximadamente 20 famílias que praticam agricultura familiar e cultivam diferentes culturas. No entorno da área, há plantações de cana-de-açúcar (cultura muito presente na região), e pequenos fragmentos de floresta que gradativamente têm sido devastados com o avanço da cultura canavieira.

\section{Coleta de drosofilídeos}

Drosofilídeos adultos foram capturados com armadilhas confeccionadas com garrafas pet, contendo isca de banana, de acordo com o modelo descrito por Tidon e Sene (1988). Em cada coleta foram utilizadas 10 armadilhas, em transecto e distanciadas pelo menos 30 metros uma da outra. e a 1,5m de altura do chão. As armadilhas permaneceram no local de coleta por três dias. As coletas foram realizadas em dois períodos sazonais distintos (chuvoso e seco). Os drosofilídeos encontrados foram transferidos para tubos contendo álcool 70\%, para posterior identificação via morfologia externa, consultando-se literatura específica, e, contagem dos espécimes coletados.

\section{Análise dos dados}

A composição de drosofilídeos foi quantificada através dos dados de abundância e riqueza de espécies. Testes de diversidade através dos índices de Shannon (H) e Simpson (1-D) foram realizados, assim como os índices de dominância (D) e riqueza (S) utilizando-se o programa Past. 


\section{Resultados e Discussão}

Foram coletados um total de 5518 indivíduos, sendo 3404 no período chuvoso e 2114 no período seco. A maior abundancia no período chuvoso, reflete o melhor desempenho reprodutivo e adaptativo em épocas mais chuvosas do que em épocas mais secas.

Dentre os indivíduos amostrados, quatro gêneros foram representados (Drosophila, Rhinoleuconphenga, Sacaptodrosofila e Zaprionus), e 16 espécies de drosofílideos. O gênero Drosophila apresentou a maior representatividade, com 13 espécies reconhecidas, o que é atribuído ao fato de que este é o gênero mais especioso dentro da família Drosophilidae. Duas espécies, Drosophila melanogaster e D. ananassae foram exclusivamente coletadas durante o período chuvoso, enquanto a estação seca apresentou três espécies exclusivas: D. mercatorum, D. pictilis e Rhinoleucophenga punctulata. Esse resultado demonstra uma suposta relação da Drosophila melanogaster e D. ananassae com umidade. As espécies coletadas exclusivamente na época seca são bem adaptadas a este período, inclusive são amostradas com freqüência em ambiente de Caatinga (Oliveira, et al 2016).

\section{Abundância e Riqueza}

Dos indivíduos coletados $83 \%$ são classificados como espécies exóticas (Drosophila ananassae, D. malerkotliana, D. melanogaster, D. simulans, Scaptodrosophila latifascaeformis e Zaprionus indianus) (Figura 1) demonstrando elevada abundância destas em ambiente cultivado, o que ocorre também em áreas abertas e urbanizadas como visto nos trabalhos de Rohde, Silva, Jucá, Montes, Garcia, 2010, Emerich, Valadão, Silva, Tidon, 2012) relatam que em ambientes cultivados existe a possibilidade de haver alta abundância de drosofílideos.

Dentre as 16 diferentes espécies amostradas, apenas 6 são consideradas exóticas, o que demonstra que, embora o estudo tenha sido realizado em um ambiente submetido a grandes interferências antrópicas de cultivo, a fauna nativa de Drosophilidae resistiu e se manteve com uma riqueza de espécies superior as exóticas. Emerich, et al (2012) também encontrou maior riqueza de nativas em seu estudo em áreas cultivadas

Figura 1. Percentual de espécies de Drosophilidae exóticas e nativas, amostradas em área cultivada do Município de Nazaré da Mata-PE.

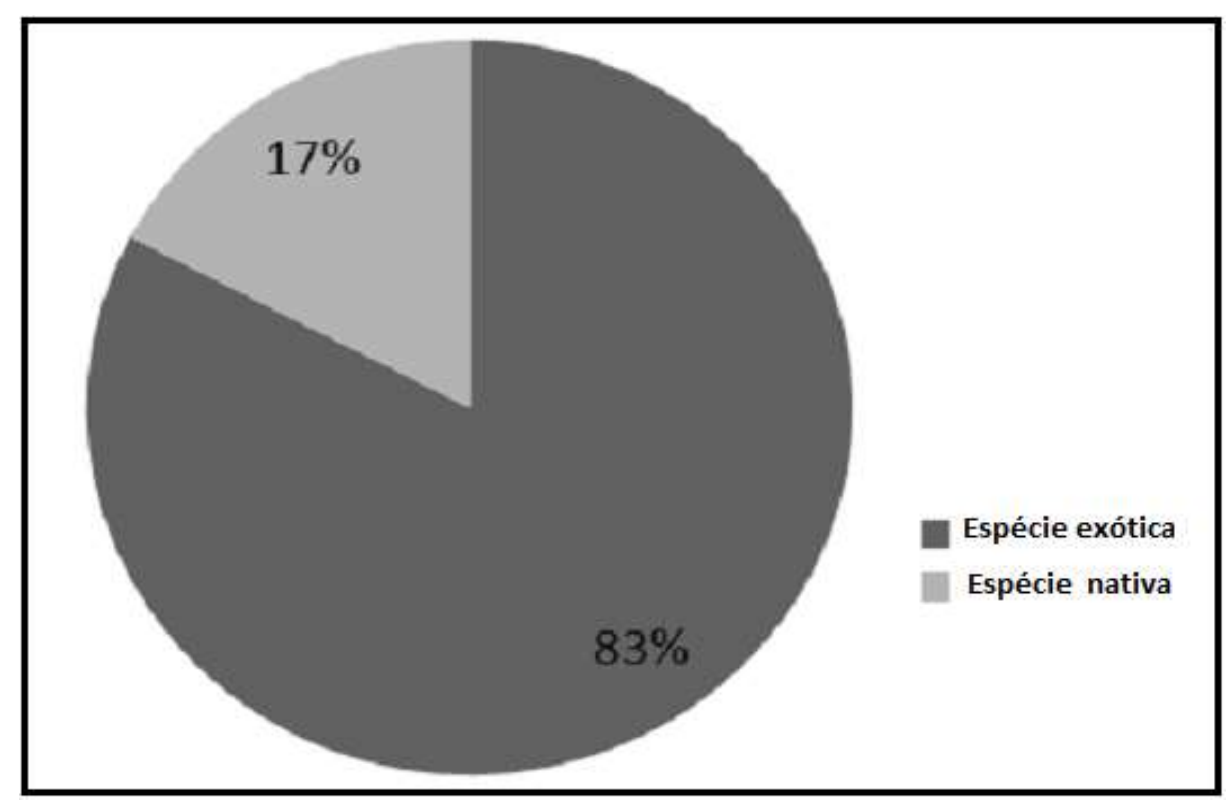

Fonte: Autores (dados da pesquisa). 
Analisando-se a Figura 2., pode-se perceber que três das espécies exóticas amostradas foram as mais abundantes neste estudo: D. malerkotliana, D. simulans e Z. indianus. Esse resultado demonstra a elevada capacidade de colonização destas espécies em áreas antropizadas. Ambientes como esses, absorvem espécies exóticas, as quais competem com as nativas e são superiores, pois estas espécies exóticas e invasoras destacam-se por apresentarem grande potencial invasor e colonizador. Esse grande potencial colonizador das espécies exóticas já foi demonstrado em diversos estudos, realizados nos mais variados ambientes Rohde et al. (2010), Yassin e David (2010), Valiati, Sofia, Silva, Garcia, Rohde, Gaiesky et al. (2005), CoutinhoSilva, Montes, OliveiraCarvalho Neto, Rohde, Garcia, (2017).

Figura 2. Ranqueamento em ordem decrescente por abundância entre as espécies amostradas em área cultivada do Município de Nazaré da Mata - PE.

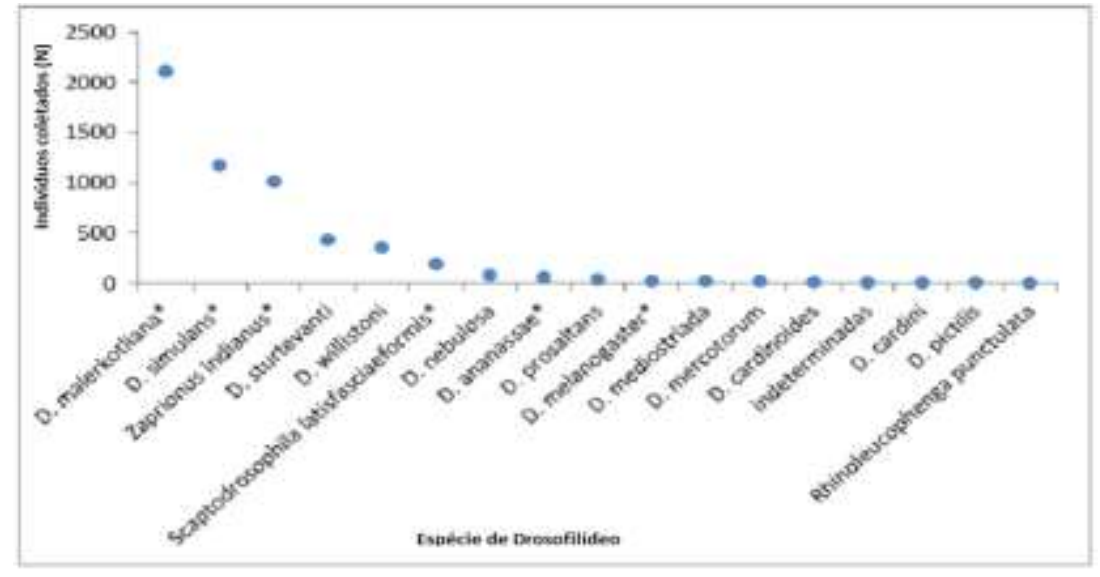

Fonte: Silva (2014).

D. malerkotliana foi introduzida em regiões de vegetação aberta no Brasil, atingindo altos índices populacionais, colonizando inicialmente bordas florestais, em seguida o interior de fragmentos, alterando as frequências das espécies nativas e dominantes (Martins, 1989). Segundo Sene et al. (1980), D. simulans foi a espécie introduzida no Neotrópico que melhor se adaptou às diferentes regiões fitogeográficas, embora seja mais abundante em áreas abertas, sendo considerada também, comum e adaptada ao ambiente de mata (Perondini et al.,1979, Saavedra et al., 1995). O potencial colonizador da Z. indianus vem sendo apontado em diversos estudos realizados no país, sobretudo em área de vegetação aberta e sobre forte ação antrópica, explorando substratos nativos e exóticos (Silva et al., 2005, Tidon et al., 2005, Castro, Valente, 2001, Vilela, 1999)

Em ambas as estações, as espécies exóticas obtiveram elevadas abundâncias, sobrepondo-se às espécies nativas (Figura 3). Estudos realizados nas florestas do sul do Brasil encontraram uma maior abundância de espécies exóticas, como a D. malerkotliana na estação com maiores índices pluviométricos (Tidon-Sklorz \& Sene, 1992, De Toni et al., 2007, Bizzo et al., 2010), em contrapartida, o padrão encontrado no Nordeste por Coutinho-Silva (2017), foi de maiores abundâncias dessas espécies apenas na estação seca.

O padrão encontrado em áreas de cultivo possui algumas semelhanças com o encontrado em áreas florestais nativas, no Sul do país (Silva, Meirelles, Soglio, Redaelli, 2004) avaliaram a população de Anastrepha spp. e C. capitata em pomares de citros, associando temperaturas mais elevadas e ocorrência de chuvas, com o aumento populacional das moscas-das-frutas, apesar de pertencerem a grupos diferentes do trabalho em estudo, são insetos e possuem um padrão de comportamento semelhante. 
Figura 3. Abundância relelativa (\%) de espécies exóticas e nativas de Drosophilidae em dois períodos sazonais distintos: Estação seca e Estação chuvosa.

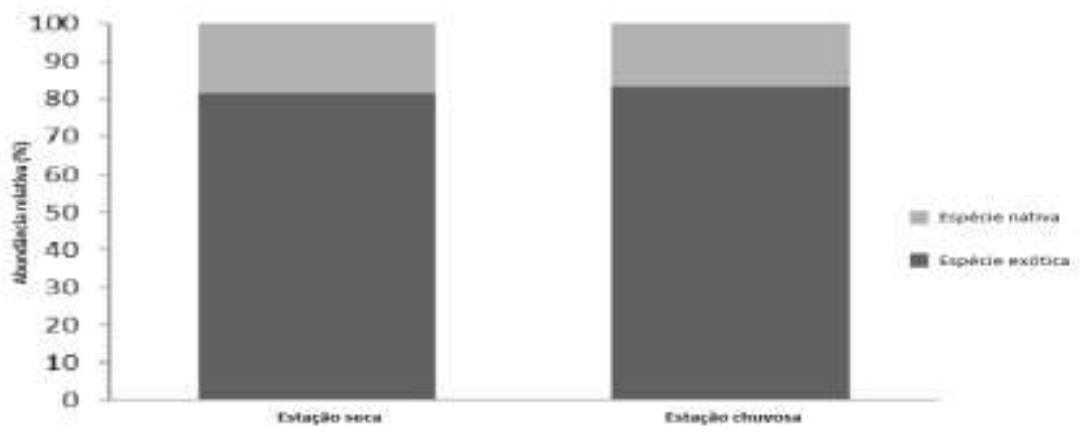

Fonte: Autores (dados da pesquisa).

Emerich et al. (2012), analisando os resultados de seu estudo nas áreas de cultivo, obteve resultados diferentes, onde a abundância de espécies nativas na região Neotropical sobrepôs às espécies exóticas a essa região. A elevada abundância de espécies nativas nesse estudo se deve ao domínio de D. cardini, espécie Neotropical amplamente distribuída em vegetações abertas da América do Sul (Vilela, Silva, Sene, 2002). a qual está bem adaptada em ambientes secos como é o caso da Savana, local estudado por esses autores.

\section{Diversidade}

A riqueza (Taxa S) de espécies e o número de indivíduos foram semelhantes entre as amostragens, no entanto, neste último houve maior abundância no período chuvoso, ao contrário do que foi visto por Coutinho-Silva (2017) em áreas florestais de Pernambuco, onde a estação chuvosa apresentou-se com maior riqueza de espécies e a estação seca apresentou maior abundância de indivíduos, especialmente espécies exóticas. A dominância foi semelhante para as diferentes estações o que faz com que os índices de diversidade (1-D e H) que medem a equitabilidade, sejam semelhantes entre as mesmas (Tabela $1)$.

Coutinho-Silva, Montes, Oliveira, Carvalho Neto, Rohde, Garcia (2017) não encontrou relação significativa entre a sazonalidade e diversidade de espécies, caso ocorrido em nosso estudo, onde a diversidade de espécies foi elevada em ambas as amostragens, e portanto, a sazonalidade não se mostrou um parâmetro distinguível em relação a composição de drosofilídeos no ambiente estudado. Esse resultado demonstra que os recursos utilizados por eles, são os mesmos e independem do período, o que faz a diversidade ser semelhante independentemente da época estudada. Esse fato tem sido comum entre os indivíduos do Filo Arthropoda, estudos realizados com outras ordens, como coleópteros, isópteros e aracnídeos, também não encontraram relação significativa entre a estação do ano e a composição de espécies (Vasconcellos, 2003, Dias, Brescovit, Couto, \& Martins, 2006, Anu, Sabu, \& Vineesh, 2009).

A não relação significativa entre a sazonalidade e abundância de insetos também é observada em áreas de cultivo, como foi analisado por Camargos, Alvarenga, Giustolin, Oliveira, Rabelo (2015), os autores estudaram moscas-da-fruta (Diptera: Tephritidae) em cafezais em Minas Gerais, e concluíram que não houve correlação significativa entre os dados populacionais e os fatores climáticos analisados, ou seja, as maiores abundâncias de insetos dípteros coincidiram em razão da maior disponibilidade de frutos maduros nos cafezais, semelhante ocorre em cultivo de pêssego (Montes, Sônia Maria Nalesso Marangoni et al. 2011), Puzzi e Orlando (1965) afirmaram que os fatores bióticos (hospedeiros e estágio de maturação dos frutos) são mais significativos na densidade populacional que os fatores abióticos (temperatura, umidade relativa e precipitação pluviométrica). Este fato foi avaliado em vários estudos, sendo sugestivo que fatores abióticos influenciem nas taxas reprodutivas das espécies (Antonini, Yasmine., Souza, Henrique, Jacobi, Claudia \& Mury Fabio B. (2005). 
Tabela 1. Analise da diversidade alpha entre a composição de Drosophilidae amostrada em área cultivada, durante estações chuvosa e seca, no município de Nazaré da Mata - PE.

\begin{tabular}{|c|c|c|}
\hline INDICE DE DIVERSIDADE & ESTAÇÃO SECA & ESTAÇÃO CHUVOSA \\
\hline Riqueza S & 13 & 214 \\
\hline Indivíduos & 3404 & 0,3384 \\
\hline Dominância (D) & 0,3038 & 0,6616 \\
\hline Simpson (1-D) & 0,6962 & 1,445 \\
\hline Shannon - (H) & 1,537 & 1,34 \\
\hline
\end{tabular}

Fonte: Autores (dados da pesquisa).

\section{Considerações Finais}

O ambiente avaliado revela o impacto da antropização sobre a comunidade de drosofílideos, apontando a elevada presença de espécies associadas à ação antrópica (exóticas).

Adicionalmente, pôde-se perceber a elevada representatividade de espécies nativas na área estudada, o que reforça a necessidade de repensar a importância da conservação dos fragmentos de Floresta Atlântica ainda existe como um reduto de espécies nativas.

No entanto, a elevada abundância de espécies exóticas remete a necessidade de atenção, uma vez que estas competem com as espécies nativas pelo uso dos recursos. Assim, a caracterização de drosofílideos mostra-se importante ferramenta no desenvolvimento de ações que visem à conservação deste ambiente, tendo em vista que medidas como estas serão importantes não só para a manutenção destes insetos, mas também para uma grande diversidade de outros organismos. Estudos futuros com amostragens em diferentes tipos de áreas cultivadas (monoculturas, cultivos orgânicos, etc.) são importantes para melhor compreensão do uso desses recursos peles drosofilídeos.

\section{Referências}

Anu, A., Sabu, T. \& Vineesh, P. J. (2009) Seasonality of litter insects and relationship with rainfall in a wet evergreen forest in south Western Ghats. Journal of Insect Science 9, 1-10.

Antonini, Y., Souza, H. G., Jacobi, C. M., \& Mury, F. B. (2005). Diversidade e comportamento dos insetos visitantes florais de Stachytarpheta glabra Cham. (Verbenaceae), em uma área de campo ferruginoso, Ouro Preto, MG. Neotrop. Entomol. 34 (4): 555-564.

Bächli, G. (2020) TaxoDros: The database on Taxonomy of Drosophilidae. http://taxodros.unizh.ch.

Bizzo, L., Gottschalk, M. S., De Toni, D. C., \& Hofmann, P. R. P. (2010). Seasonal dynamics of a drosophilid (Diptera) assemblage and its potencial as bioindicator in open environments. Iheringia Ser. Zool. 100, 185-191.

Camargo, A. J. A. de. (2001). Diversidade de insetos em áreas cultivadas e reserva legal: considerações e recomendações. Embrapa Cerrados. 27p.

Camargos, M. G., Alvarenga, C. D., Giustolin, T. A., Oliveira, P. C. C., \& Rabelo, M. M. (2015). Moscas-das-frutas (Diptera: Tephritidae) em cafezais irrigados no norte de Minas Gerais. Coffee Science, 10, 28-37.

Castro, F. L., \& Valente, V. L. S. (2001). Zapriounus indianusis invading Drosophilid communites in the southern Brazilian city of Porto Alegre. Drosophila Information Service. 84, 15-17.

Coutinho-Silva, R. D., Montes, M. A., Oliveira, G. F., Carvalho Neto, F. G., Rohde, C, \& Garcia, A. C. L. (2017). Effects of seasonality on drosophilids (Insecta, Diptera) in the northern part of the Atlantic Forest, Brazil. Bulletin Of Entomological Research, 107, 634-644.

De Toni, D.C., Gottschalk, M.S., Cordeiro, J., Hofmann, P.P.R., \& Valente, V.L.S. (2007). Study of the Drosophilidae (Diptera) communities on Atlantic Forest Islands of Santa Catarina State, Brazil. Neotrop. Entomol. 36, 356-375.

Dias, S. C., Brescovit, A. D., Couto, E. C. G. \& Martins, C.F. (2006) Species richness and seasonality of spiders (Arachnida, Araneae) in an urban Atlantic Forest fragment in Northeastern Brazil. Urban Ecosystems 9, 323-335.

Emerich, P. P., Valadão, H., Silva, J. R. V. P., \& Tidon, R. (2012). High Abundance of Neotropical Drosophilids (Diptera: Drosophilidae) in Four Cultivated Areas of Central Brazil. Instituto de Ciências Biológicas-PPG Ecologia, Univ de Brasília, Brasília, DF, Brasil. Neotrop Entomol. 41, 83-88.

Garcia, C. F, Hochmüller, C. J. C, Valente, V. L. S., \& Schmitz, H. J. (2012). Drosophilid Assemblages at Different Urbanization Levels in the City of Porto Alegre, State of Rio Grande do Sul, Southern Brazil. Neotropical Entomology (Impresso), 41, 1-41. 
Gottscalk, M. S., Hofmann, P. R. P., \& Valente, V. L. S. (2008). Díptera, Drosophilidae: historical occurrence in Brazil. Checklist. 4, $485-518$.

INPE (2010, 26 de maio). Dados do Atlas dos Remanescentes Florestais da Mata Atlântica revelam desmate de ao menos 20.867 hectares nos últimos dois anos. http://www.inpe.br/noticias/noticia.php?Cod_Noticia=2199.

Martins, M. B. (1989) Invasão de fragmentos florestais por espécies oportunistas de Drosophila (Diptera, Drosophilidae). Acta Amazônica. $19,265-271$.

Montes, S. M. N. M., Rafael, A., Bolívia, A., C., Santos, P., C. (2011). Dinâmica populacional e incidência de moscas-das-frutas e parasitoides em cultivares de pessegueiros (Prunus Persica L. Batsch) no município de presidente prudente-sp. Revista Brasileira de Fruticultura. 33(2), 402-411. <https://doi.org/10.1590/S0100-29452011005000052>.

Oliveira, G. F., Rohde, C. Garcia, A. C. L., Montes, M. A., \& Valente, V. L. S. (2016). Contributions of dryland forest (Caatinga) to species composition, richness and diversity of Drosophilidae. Neotropical Entomology (Impresso), 45, 537-547.

Perondini, A. L. P., Sene, F. M., \& Mori, L. (1979). The pattern and polymorphism of some Drosophila simulans esterases in Brazil. Egyptian Journal of Genetics and Cytology. v.8, pp.263-268.

Powell JR. (1997). Progress and prospects in evolutionary biology: the Drosophila Model. Oxford University Press, New York.

Puzzi, D., \& Orlando, A. (1965) Estudos sobre a ecologia das moscas-das-frutas (Tephritidae) no Estado de São Paulo, visando o controle racional da praga. Arq. lnst. Biol., 32(1):9-22.

Rêgo, G.M., \& Hoeflich, V.A. (2001). Contribuição da pesquisa florestal para um ecossistema em extinção: Floresta Atlântica do Nordeste do Brasil. Aracaju: Embrapa Tabuleiros Costeiros. 80p. (Embrapa Tabuleiros Costeiros. Documentos.

Rohde, C., Silva, D.M.I.O., Jucá, J. C. L. A., Montes, M.A., \& Garcia, A. C. L. (2010). Espécies invasoras da família Drosophilidae (Diptera, Insecta) em ambientes da Caatinga de Pernambuco. An. Acad. Pern. Ciênc. Agron. 7, 227-240.

Saavedra, C. C. R., Callegari-Jacques, S. M., Napp, M., Valente, V. L. S. (1995). A descriptive and analytical study of four Neotropical drosophilid communities. Journal of Zoological Systematics and Evolutionary Research. 33, 62-74.

Schmitz, H. J., \& Valente, V. L. S. (2019). The flower flies and the unknown diversity of Drosophilidae (Diptera): a biodiversity inventory in the Brazilian fauna. Papéis Avulsos De Zoologia. 59, 1-15.

Sene, F. M., Val, F.C., Vilela, C. R., \& Pereira, M. A. Q. R. (1980). Preliminary date on the geographical disttribution of Drosophila species within morphoclimatic domains of Brazil. Paps. Av. Zool. 33(22), 315-326.

Silva, M S. (2014). Caracterização da Fauna de Drosophilidae (Insecta, Diptera) em ambiente devastado de Floresta Atlântica na Zona da Mata Norte de Pernambuco. TCC, Universidade Federal de Pernambuco. 40p.

Silva, F. F. da, Meirelles, R N., Soglio, F. K. D., \& Redaelli, L. R. (2004). Influência de variáveis climáticas na ocorrência de moscas-das-frutas (Diptera:Tephritidae) em pomares de citros, na região do Vale do Rio Caí, Rio Grande do Sul. In: Congresso Brasileiro De Entomologia, Gramado. Anais... Gramado: SEB. p.652

Silva, N. M., Fantinel, C. C., Valente, V. L. S., \& Andvaliati V. H. (2005). Population dynamics of the invasive species Zaprionus indianus (Gupta) (Diptera: Drosophilidae) in communities of Porto Alegre city, Southern of Brazil. Neotropical Entomology. 34(3), 363-374.

SOS Mata Atlantica. (2020). Instituto Nacional De Pesquisas Espaciais. Atlas dos remanescentes florestais da mata atlântica período 2018 -2019 Fundação SOS Mata Atlântica, pp.33-36.

SOS Mata Atlantica. (2002). Instituto Nacional De Pesquisas Espaciais. Atlas dos remanescentes florestais da Mata Atlântica: período 1995-2000. Fundação SOS Mata Atlântica, pp.42-43.

Tidon, R., Leite, D. F., Ferreira, L., \& Leão, B. F. D. (2005). Drosofilídeos (Diptera, Drosphilidae) do Cerrado. In: Scariot, A., Felfili, J. \& Silva, J. C. S. E. (Eds). Ecologia e Biodiversidade do Cerrado. Ministério do Meio Ambiente, Brasília, Brasil, 337-352.

Tidon, R., \& Sene, F. M. (1988). A trap that retains and keeps Drosophlaalive. Drosophila Information Service, 67, 88-89.

Tidon-Sklorz, R., \& Sene, F. M. (1992). Vertical and temporal distribution of Drosophila (Diptera, Drosophilidae) species in a wooded area in the state of São Paulo, Brazil. Revista Brasileira de Biologia. 52, 331-317.

Triplehorn, C. A., Johnson, N. F. (2011) Estudo dos insetos. Cengage Learning. Tradução de Borror and Delong's introduction to the study of insects. p.816.

Valiati, V. H., Sofia, T., Silva, N. M., Garcia, A. C. L, Rohde, C., \& Gaiesky, V. L. S. V. (2005). Colonização, competição e coexistência: insetos como modelo de invasões biológicas. Logos 16, 13-23.

Vasconcellos, A. (2003) Ecologia e biodiversidade de cupins em remanescentes de Mata Atlântica do Nordeste Brasileiro. Ph.D. Thesis. Universidade Federal da Paraíba, Brazil.

Vilela, C. R., Silva, A. F. G., \& Sene, F. M. (2002). Preliminary data on the geographical distribution of Drosophila species within morphoclimatic domains of Brazil.III. The cardini group. Revista Brasileira de Entomologia, 46, 139-148.

Vilela, C. R. (1999). Is Zaprionus indianus Gupta, 1970 (Diptera, Drosophilidae) currently colonizing the Neotropical Region?

Yassin, A. \& David J. R. (2010). Revision of the Afrotropical species of Zaprionus (Diptera: Drosophilidae), with descriptions of two new species and notes on internal reproductive structures and immature stages. Zookeys. 51, 33-72. 